\title{
La promoción del trabajo decente a través del principio cooperativo de educación, formación e información
}

(The promotion of decent work through the cooperative principle concerning education, training and information)

\author{
Josune López Rodríguez² \\ Universidad de Deusto (España)
}

Sumario: 1. Introducción. 2. Concepto de trabajo decente. 3. Las cooperativas como entidades adecuadas para promover el trabajo decente. 4. La promoción del trabajo decente a través del principio cooperativo de educación, formación e información. 4.1. Los principios cooperativos: alcance del quinto principio cooperativo. 4.2. El quinto principio cooperativo como instrumento para promover el trabajo decente. 5. A modo de conclusión. 6. Bibliografía.

Summary: 1. Introduction. 2. Concept of decent work. 3. Cooperatives as appropriate entities to promote decent work. 4 . The promotion of decent work through the cooperative principle regarding education, training and information. 4.1. The cooperative principles: the scope of the fifth cooperative principle. 4.2. The fifth cooperative principle as a tool to promote decent work. 5. In conclusion. 6. Bibliography.

1 Este trabajo forma parte del Proyecto de Investigación titulado «Las cooperativas como instrumento de política de empleo ante los nuevos retos del mundo del trabajo» (RTI2018-097715-B-I00). El proyecto ha sido financiado por el Ministerio de Ciencia, Innovación y Universidad del Gobierno español, la Agencia Estatal de Investigación y el Fondo Europeo de Desarrollo Regional de la Unión Europea, en el marco de la Convocatoria correspondiente a 2018 de Proyectos de I+D+i «Retos de investigación» del Programa Estatal de I+D+i orientado a los retos de la sociedad.

Además, este trabajo está basado en la ponencia titulada «El principio cooperativo de educación, formación e información como herramienta para promover el trabajo digno» y presentada en el Congreso «El principio de educación, formación e información de las cooperativas. Consecuencias jurídicas y económicas derivadas de su aplicación práctica», organizado por la Asociación Internacional de Derecho Cooperativo, la Academia Vasca de Derecho y la Facultad de Derecho de la Universidad de Deusto, y celebrado en la Universidad de Deusto, Bilbao, los días 13 y 14 de noviembre de 2019.

2 josunelopezrodriguez@deusto.es 
Resumen: La finalidad de este artículo consiste en presentar el quinto principio cooperativo de educación, formación e información como un instrumento óptimo para promover el trabajo decente en las cooperativas. Precisamente, existe en la actualidad un déficit global de trabajo decente que requiere respuestas eficaces y urgentes. A tal fin, primero, se examinará brevemente el alcance del concepto de trabajo decente. Después, se procederá a resaltar la relevancia de las cooperativas como entidades apropiadas para fomentar el trabajo decente. Y, con base en este análisis, se presentará el quinto principio cooperativo como herramienta para promover el trabajo decente en el ámbito de las cooperativas.

Palabras clave: Trabajo decente; cooperativas; quinto principio cooperativo; educación, formación e información.

Abstract: The purpose of this paper lies in presenting the fifth cooperative principle concerning education, training and information as an optimal tool to promote decent work within cooperatives. In fact, the current global decent work deficit requires efficient and urgent answers. To that end, first, we will briefly study the scope of the concept of decent work. After that, we will highlight the significance of cooperatives as appropriate entities to promote decent work. In addition, based on this analysis, we will present the fifth cooperative principle as a tool to promote decent work in the field of cooperatives.

Keywords: Decent work; cooperatives; fifth cooperative principle; education, training and information. 
«Educar es lo mismo que poner un motor a una barca... Hay que medir, pensar, equilibrar...

y poner todo en marcha.

Pero para eso, uno tiene que llevar en el alma un poco de marino... un poco de pirata... un poco de poeta... y un kilo y medio de paciencia concentrada (...)»

Gabriel Celaya

\section{Introducción}

El principal objetivo de este artículo radica en presentar el quinto principio cooperativo, esto es, el principio cooperativo de educación, formación e información, como una herramienta o un instrumento óptimo para promover el trabajo decente en el ámbito del cooperativismo.

En el contexto actual, el estudio de esta materia resulta de suma importancia en tanto en cuanto la realización del trabajo decente constituye uno de los Objetivos incluidos en la Agenda 2030 para el Desarrollo Sostenible, aprobada mediante la Resolución de la Asamblea General de las Naciones Unidas de 25 de septiembre de 2015. Más aún, las alarmantes consecuencias que la pandemia de la COVID-19 está acarreando en el ámbito del trabajo, en especial, la masiva destrucción de empleos y la constante precarización laboral, ponen de manifiesto la imperiosa necesidad de crear y mantener empleos de calidad.

En concreto, el Objetivo 8 de la mencionada Agenda 2030 tiene como propósito "promover el crecimiento económico inclusivo y sostenible, el empleo y el trabajo decente para todos», al entender que "un crecimiento inclusivo y sostenido puede impulsar el progreso, crear empleos decentes para todos y mejorar los estándares de vida». Pues bien, el establecimiento de este Objetivo se basa en el hecho de que existe actualmente un déficit global de trabajo decente. Por un lado, existe un déficit de trabajo caracterizado por las altas tasas de desempleo a nivel mundial, una situación que afecta especialmente a los jóvenes y 
que se ha visto agravada con la pandemia de la COVID-19. Y, por otro lado, existe un déficit de calidad en el trabajo, ya que no todos los trabajos existentes son trabajos de calidad.

Así, la ausencia generalizada de trabajo decente acentúa la desigualdad y las situaciones de exclusión social y, al mismo tiempo, afecta negativamente a la economía. Esta preocupante situación acarrea el desafío mundial de crear empleos decentes, empleos de calidad, ello en aras de garantizar un desarrollo económico y social sostenido, inclusivo y sostenible.

Y para conseguir el reto que supone la materialización del trabajo decente, la educación, la formación y la información cobran, sin duda, especial relevancia. De este modo, la educación, la formación y la información, que contribuyen a mejorar la calidad y la eficacia de los puestos de trabajo, deben constituir herramientas esenciales para reducir el déficit de trabajo decente. Además, estas herramientas deben emplearse especialmente en el ámbito de las cooperativas, en el que la persona se sitúa en el centro de la actividad.

Con base en estos antecedentes, y para lograr el objetivo del presente artículo, primero, se estudiará brevemente el alcance del concepto de trabajo decente. Una vez sentada esta base conceptual, se procederá a resaltar la relevancia de las cooperativas como entidades apropiadas para fomentar el trabajo decente. Y, a partir de este análisis, se presentará el quinto principio cooperativo como promotor del trabajo decente en el seno de las cooperativas.

\section{Concepto de trabajo decente}

La expresión «trabajo decente» ${ }^{3}$ fue definida por primera vez en la Memoria del Director General de la Organización Internacional del Tra-

3 Antes de examinar el significado de esta expresión, conviene hacer un breve comentario acerca de los términos empleados. Precisamente, la expresión original inglesa, "decent work», ha sido traducida al español de manera literal como "trabajo decente», lo que ha dado lugar a interpretaciones erróneas sobre su verdadero alcance. En efecto, el adjetivo empleado en la versión española, «decente», denota "una carga moral extraordinaria, ya que en inglés no es de semejante categoría» (Bordas Martínez, J. y Pinilla García, 2020, 81). Así, la expresión inglesa «decent work» viene a significar «trabajo adecuado o, mejor aún, trabajo digno; de tal manera que lo indecente no es el trabajo realizado, sino la forma de ejecutarlo» (Bordas Martínez, J. y Pinilla García, 2020, 81). Además, cabe señalar que el adjetivo «decente» no tiene una tradición en el ordenamiento jurídico español y no mantiene un vínculo con los principios propios del ordenamiento jurídico interno. Por el contrario, la expresión «trabajo digno» sí que está 
bajo a la 87. a reunión de la Conferencia Internacional del Trabajo, celebrada en 1999. Se trata de un concepto que surge como respuesta a la globalización y a la crisis y que pretende aunar en solo dos palabras un conjunto de principios y derechos relacionados con los conceptos de dignidad humana, libertad, seguridad y equidad (Egger y Sengenberger, 2001, 37).

Posteriormente, la Declaración de la Organización Internacional del Trabajo sobre la justicia social para una globalización equitativa, adoptada en 2008, institucionalizó este concepto como «el principio organizativo de las tareas» de la Organización y consideró que debía ser el elemento central de las políticas económicas y sociales (Gil y Gil, 2020, 142). En plena crisis económica, el Pacto Mundial para el Empleo, adoptado por la Conferencia Internacional del Trabajo el 19 de junio de 2009, determinó que el «objetivo del empleo pleno y productivo y el trabajo decente debe ponerse en el centro de las respuestas a la crisis». Y, recientemente, la Declaración del Centenario para el futuro del trabajo, aprobada en 2019 por la Organización Internacional del Trabajo con ocasión de su centenario, ha confirmado que resulta indispensable "actuar urgentemente para aprovechar las oportunidades y afrontar los retos a fin de construir un futuro del trabajo justo, inclusivo y seguro con empleo pleno, productivo y libremente elegido y trabajo decente para todos».

Pues bien, a grandes rasgos, el trabajo decente constituye «un concepto ético-jurídico y un marco integrador de los pilares del mandado constitucional» de la Organización Internacional del Trabajo: la promoción del empleo, la protección de los derechos en el trabajo, la extensión de la protección social, el fomento del diálogo social, y el respeto de la igualdad de oportunidades y trato para las mujeres y los hombres (Gil y Gil, 2017, 3). Y, más concretamente, esta expresión se refiere al «trabajo productivo en condiciones de libertad, equidad, seguridad y dignidad, en el cual los derechos son protegidos y que cuenta con remuneración adecuada y protección social» (Conferencia Internacional del Trabajo, 1999).

relacionada con un valor superior del ordenamiento jurídico y apela a un derecho fundamental de la persona reconocido expresamente en el texto constitucional, a saber, la dignidad (Cruz Villalón, 2019, 243). De este modo, teniendo en cuenta que la dignidad constituye un concepto jurídico enraizado en el ordenamiento jurídico interno y que el término "decente», en cambio, se concibe como una referencia moral o política, se considera que, desde una perspectiva jurídica, la expresión más adecuada resulta la de «trabajo digno» (Cruz Villalón, 2019, 243). No obstante estas consideraciones, en el presente escrito se utilizará la expresión «trabajo decente», por ser este el calificativo oficial empleado por la Organización Internacional del Trabajo. 
Así, la noción de trabajo decente adquiere cuatro dimensiones principales, esto es: el trabajo; los derechos fundamentales en el trabajo; la protección social; y la representación y el diálogo (Ghai, 2003, 125 y Rodgers, 2002, 14-16).

En primer lugar, el trabajo decente engloba todas las clases de trabajo. Así, la noción de trabajo decente comprende tanto a los trabajadores asalariados (de la economía formal e informal) como a los trabajadores autónomos.

En segundo lugar, el trabajo decente implica la promoción y el cumplimiento de los derechos fundamentales en el trabajo, derechos que están recogidos en los ocho convenios fundamentales de la Organización Internacional del Trabajo, a saber: la libertad de asociación, la libertad sindical y el derecho a la negociación colectiva; la abolición del trabajo forzoso u obligatorio; la erradicación del trabajo infantil; y la eliminación de toda forma de discriminación en materia de empleo y ocupación.

En tercer lugar, la seguridad social y la seguridad de ingresos constituyen otro de los elementos esenciales del trabajo decente, si bien es cierto que estos conceptos dependen de la capacidad y el nivel de desarrollo de cada sociedad.

Y, en cuarto lugar, respecto de la representación y el diálogo, la idea de trabajo decente supone que los trabajadores deben ejercer el derecho a expresar sus opiniones, a defender sus intereses y a entablar negociaciones con los empresarios y con las autoridades sobre las cuestiones relacionadas con la actividad laboral.

En síntesis, el trabajo decente constituye un concepto flexible mediante el cual se hace alusión a los derechos y principios socio-laborales universales y, al mismo tiempo, se reflejan las circunstancias específicas de cada país (Lozano Lares, 2016, 13). Precisamente, el trabajo decente es un objetivo, una meta, que debe constituir el suelo mínimo a respetar por los países, pero que su interpretación concreta y su aplicación dependerán de las condiciones de cada sociedad.

\section{Las cooperativas como entidades adecuadas para promover el trabajo decente}

Antes de estudiar el alcance del quinto principio cooperativo y defender que el mismo constituye un instrumento óptimo para promover el trabajo decente, resulta necesario presentar las sociedades cooperativas como entidades apropiadas para fomentar el trabajo decente. 
En términos generales, según la definición aportada por la Declaración de la Alianza Cooperativa Internacional sobre la Identidad Cooperativa, adoptada en 1995, la cooperativa es «una asociación autónoma de personas que se agrupan voluntariamente para satisfacer sus necesidades y aspiraciones comunes de carácter económico, social y cultural mediante una empresa de propiedad conjunta y gestionada democráticamente». Para lograr sus propósitos, la cooperativa debe actuar sobre la base de una serie de principios, a saber: la adhesión voluntaria y abierta; la gestión democrática de las personas socias; la participación económica de las personas socias; la autonomía e independencia; la educación, formación e información; la cooperación entre cooperativas; y el interés por la comunidad (Morgado Panadero, 2006, 4345). Estos principios llevan a las cooperativas «a una constante preocupación y atención por su entorno, tanto por las personas como por el mismo medio ambiente en el que están emplazadas y desarrollan su actividad» (Martínez Charterina, 2013, 196).

En el ordenamiento jurídico español, la Constitución Española ${ }^{4}$ se refiere expresamente al fomento del cooperativismo en su artículo 129.2 , en el que dispone que «los poderes públicos promoverán eficazmente las diversas formas de participación en la empresa y fomentarán, mediante una legislación adecuada, las sociedades cooperativas».

Más concretamente, el artículo 1.1 de la Ley 27/1999, de 16 de julio, de Cooperativas 5 , define la cooperativa como «una sociedad constituida por personas que se asocian, en régimen de libre adhesión y baja voluntaria, para la realización de actividades empresariales, encaminadas a satisfacer sus necesidades y aspiraciones económicas y sociales, con estructura y funcionamiento democrático, conforme a los principios formulados por la alianza cooperativa internacional, en los términos resultantes de la presente Ley». Además, la Ley 5/2011, de 29 de marzo, de Economía Social ${ }^{6}$, establece que las cooperativas forman parte de la Economía Social (cfr. artículo 5.1). A este último respecto, cabe señalar que la Economía Social es un sector en el que lo característico «no es tanto la actividad económica que desarrolla, como el modo de desarrollarla y el fin perseguido con la misma, esto es, bien el interés de servicio a sus socios, bien el interés general, o ambos» (García Ninet, 2014, 42).

\footnotetext{
4 BOE núm. 311, de 29 de diciembre de 1978.

5 BOE núm. 170, de 17 de julio de 1999.

6 BOE núm. 76, de 30 de marzo de 2011.
} 
En suma, las cooperativas son empresas enmarcadas en la Economía Social en las que «un grupo de personas comparte una o varias necesidades y decide satisfacerlas directamente por ellas mismas, al margen de las diversas oportunidades que les pueda prestar el entorno en el que viven, a través de una empresa económica» (Martínez Charterina, 2015a, 107) y que se caracterizan «por ser una fuerza para el cambio social, y contribuir a la transformación de la estructura socioeconómica del país para el logro de una mayor igualdad y justicia» (Rodríguez González, 2018, 5).

Con base en estas ideas, las cooperativas han sido concebidas como una fórmula empresarial adecuada para afrontar las crisis económicas y para crear empleo de calidad, principalmente, por la «mayor corresponsabilidad de los trabajadores en la toma de decisiones para la flexibilidad interna y calidad del empleo» (Calderón, B. y Calderón, 2012, 54). Ahora bien, es importante puntualizar que las cooperativas no deben considerarse únicamente como alternativas empresariales ante las situaciones de recesión económica, sino como "verdaderos motores del cambio, un cambio necesario para superar las crecientes desigualdades que los paradigmas económicos tradicionales han conllevado, al margen de las situaciones de crisis» (Gaminde Egia, 2018, 39).

A partir de lo dicho, en tanto en cuanto las cooperativas son entidades transformadoras que contribuyen al cambio social y que tienen a la persona como pilar fundamental, se cree que las mismas deben ser un referente en la promoción del trabajo decente. Esta idea ha quedado reflejada en distintos textos jurídicos, tanto internacionales como internos.

En el ámbito jurídico internacional, el Pacto Mundial para el Empleo, de 2009, destaca la importancia de la cooperación para secundar la transformación de la economía en aras de hacer realidad el trabajo decente (cfr. apartado 21.3). En otras palabras, en el texto queda patente el estrecho vínculo existente entre la cooperación, lo que puede traducirse en la fórmula cooperativa, y la realización del trabajo decente.

En 2002, la Organización Internacional del Trabajo adoptó la Recomendación número 193, sobre la promoción de las cooperativas, aunque se trata de un texto sin valor normativo (Bengoetxea Alkorta, 2016, 10). En el Preámbulo de dicha Recomendación, se pondera la importancia de las cooperativas para la creación de empleos. Igualmente, se dispone en el Preámbulo que «las cooperativas, en sus diversas formas, promueven la más completa participación de toda la población en el desarrollo económico y social». Y se recuerda, asimismo, 
que «el logro del trabajo decente para los trabajadores, dondequiera que se encuentren, es un objetivo primordial de la Organización Internacional del Trabajo». Más concretamente, en el texto de la Recomendación se insta a los Estados a «adoptar medidas para promover el potencial de las cooperativas en todos los países, independientemente de su nivel de desarrollo, con el fin de ayudarlas a ellas y a sus socios a: [entre otros] (a) crear y desarrollar actividades generadoras de ingresos y empleo decente y sostenible». En esta última sugerencia se reconoce expresamente la idoneidad de las entidades cooperativas para crear y mantener empleos decentes.

En 2018, la Alianza Cooperativa Internacional adoptó la Declaración sobre el trabajo digno y contra el acoso. En esta Declaración, se dispone que «el movimiento cooperativo comparte el enfoque de la Agenda 2030 para el desarrollo sostenible respecto a un mundo [...] donde todo el mundo pueda disfrutar de un trabajo digno y beneficiarse de un crecimiento económico sostenido, inclusivo y sostenible». Y se establece, además, que la Alianza Cooperativa Internacional, en su calidad de portavoz internacional del movimiento cooperativo, "se compromete a respetar y promover los postulados fundamentales del trabajo digno y a trabajar de manera diligente para apoyarlos». Estas disposiciones denotan un claro compromiso del referente internacional del cooperativismo con la promoción del trabajo decente.

En junio del año 2019, la Organización Internacional del Trabajo aprobó la Declaración del Centenario para el futuro del trabajo, en la cual se compromete a promover la Economía Social y, en particular, las cooperativas, todo ello con el fin de generar trabajo decente, empleo productivo y mejores niveles de vida. Ciertamente, la Organización Internacional del Trabajo reconoce que el mundo laboral está siendo objeto de importantes transformaciones y que, en este contexto, la cooperación, ejercida a través de las cooperativas y de otras entidades de la Economía Social, puede aportar respuestas adecuadas a dichos cambios.

En íntima conexión con la Declaración del Centenario para el futuro del trabajo, la Comisión Mundial sobre el Futuro del Trabajo publicó en 2019 el informe "Trabajar para un futuro más prometedor». En esencia, este informe constituye un programa centrado en las personas que se asienta en tres ejes de actuación orientados a generar crecimiento, igualdad y sostenibilidad para las generaciones presentes y futuras. Concretamente, el tercero de los ejes radica en incrementar la inversión en trabajo decente y sostenible, para lo cual se propone, entre otras medidas, transformar las economías. De esta manera, la Comisión recomienda la adopción de incentivos para promover las inver- 
siones en áreas clave que promuevan el trabajo decente y sostenible, así como la inversión en esferas de la economía que revisten una importancia estratégica para satisfacer necesidades mundiales ineludibles y aprovechar oportunidades de trabajo decente y sostenible. Sin duda alguna, en este marco "se sitúa el fomento de la economía social, pues esta se caracteriza por priorizar las personas y el fin social sobre el capital y los beneficios económicos» (Canalda Criado, 2019, 78).

En el ámbito de la Unión Europea, la Resolución del Parlamento Europeo, de 19 de febrero de 2009, sobre Economía Social, subraya que la Economía Social, en la cual se enmarcan las cooperativas, «desempeña un papel esencial en la economía europea al permitir la creación de empleos de calidad». Asimismo, en la Resolución se reconoce que la Economía Social es un agente clave para conseguir los objetivos delimitados en la Estrategia de Lisboa. A este respecto, se señala que la Economía Social "contribuye a rectificar tres desequilibrios fundamentales del mercado de trabajo: el desempleo, la inestabilidad en el trabajo y la exclusión de los desempleados tanto en el plano social como en el mercado laboral» y que, además, «tiene una función en la mejora de la empleabilidad y que crea empleos que normalmente no se deslocalizan». Más aún, se puntualiza en el texto que «los empleadores en la Economía Social son importantes agentes de la reinserción», a la vez que se reconocen los esfuerzos de los mismos «por crear y mantener empleos de alta calidad, buenos y estables, y de invertir en los trabajadores».

$Y$, en lo que al marco jurídico interno se refiere, tal y como se ha explicado previamente, el artículo 129.2 de la Constitución española requiere a los poderes públicos el fomento de las sociedades cooperativas. En conexión con estos mandatos constitucionales, la Ley 5/2011, de 29 de marzo, de Economía Social, dispone que las cooperativas son entidades de Economía Social y, como tales, deben actuar con base en una serie de principios orientadores, entre los que cabe destacar especialmente el descrito en el apartado c), que hace alusión expresa a la «generación de empleo estable y de calidad».

A partir de lo expuesto, cabe concluir que existe un convencimiento, tanto a nivel internacional como interno, acerca del importante papel que pueden y deben desempeñar las cooperativas en la promoción del trabajo decente. Precisamente, las cooperativas sitúan a las personas en el centro de su actividad; basan su labor en valores como la democracia, la igualdad, la equidad y la solidaridad; y están comprometidas con su entorno, con el desarrollo de la comunidad y con el bienestar de las personas. Estos factores demuestran que las cooperativas son entidades idóneas para generar trabajo decente. 
Empero, es importante señalar que para que las cooperativas puedan llevar a cabo de manera efectiva esta función, se necesitan políticas eficaces y se requiere invertir en educación y formación. Además, en esta labor, es necesario prevenir la proliferación de las falsas cooperativas que, al fin y a la postre, acaban desvirtuando la relevancia del movimiento cooperativo y de sus valores y principios. Ciertamente, la Recomendación número 193 de la Organización Internacional del Trabajo insiste en esta última idea al determinar que las políticas nacionales deberían, entre otras cuestiones, «velar por que no se puedan crear o utilizar cooperativas para evadir la legislación del trabajo ni ello sirva para establecer relaciones de trabajo encubiertas, y luchar contra las seudo-cooperativas, que violan los derechos de los trabajadores, velando por que la legislación del trabajo se aplique en todas las empresas» [cfr. apartado 8.1.b)].

\section{La promoción del trabajo decente a través del principio cooperativo de educación, formación e información}

\subsection{Los principios cooperativos: alcance del quinto principio cooperativo}

El trabajo diario de las cooperativas debe fundamentarse en el respeto y cumplimiento de los valores y principios cooperativos (Atxabal Rada, 2015, 168).

Sobre esta cuestión, la Declaración de la Alianza Cooperativa Internacional enuncia una serie de valores operativos y éticos que deben regir las actuaciones de las cooperativas y de los socios cooperativistas. Concretamente, "las cooperativas se basan en los valores de autoayuda, responsabilidad personal, democracia, igualdad, equidad y solidaridad» y "los socios integrantes de la cooperativa creen en los valores éticos de la honradez, la transparencia, la responsabilidad social y la preocupación por los demás».

Y para materializar y poner en práctica los valores mencionados, las cooperativas deben cumplir necesariamente los siete principios recogidos en la Declaración sobre la Identidad Cooperativa, a saber: (1) la adhesión voluntaria y abierta; (2) la gestión democrática por parte de las personas socias; (3) la participación económica de las personas socias; (4) la autonomía e independencia; (5) la educación, formación e información; (6) la cooperación entre cooperativas; y (7) el interés por la comunidad (Morgado Panadero, 2006, 43-45).

A grandes rasgos, los principios cooperativos son «auténticos pilares y normas fundamentales que configuran la organización, funcio- 
namiento y naturaleza cooperativa» (Salinas Ramos, 1995, 179) y que «determinan las cualidades esenciales que hacen que las cooperativas sean diferentes a los otros tipos de empresa y que el movimiento cooperativo sea valioso» (Gadea Soler, 2012, 10). Así, estos principios, formulados por la Alianza Cooperativa Internacional, se configuran como «criterios orientadores, no sólo de la actuación del legislador en materia de cooperativas, sino también de la autonomía de la voluntad de los particulares en orden a establecer nuevos pactos no previstos por la Ley» (Alfonso Sánchez, 2015, 10-11).

En concreto, el quinto principio cooperativo, esto es, el principio de educación, formación e información, es un principio "característico y singular» de las sociedades cooperativas (Macías Ruano, 2015, 7). En el ámbito jurídico interno, la Ley 5/2011, de 29 de marzo, de la Economía Social, no recoge este principio entre los principios orientadores de las entidades de Economía Social; sin embargo, cabe advertir que este principio cooperativo tiene «cierto reflejo tangencial» en el tercer principio orientador de las entidades de Economía Social en lo que concierne a la generación de empleo estable y de calidad (Macías Ruano, 2015, 7).

Pues bien, en virtud del quinto principio cooperativo, conocido como la «regla de oro del cooperativismo» (Martínez Charterina, 2015b, 38), las cooperativas «proporcionan educación y formación a los socios, a los representantes elegidos, a los directivos y a los empleados para que puedan contribuir de forma eficaz al desarrollo de sus cooperativas. Informan al gran público, especialmente a los jóvenes y a los líderes de opinión, de la naturaleza y beneficios de la cooperación».

Este principio tiene una doble vertiente ad intra y ad extra de la cooperativa. Desde una perspectiva interna, la formación cooperativa está relacionada directamente con la forma de gestión y gobierno de la cooperativa y, por eso, se dirige a las personas que forman parte de la misma (directivos, socios, trabajadores). Mediante esta formación interna se consigue materializar, además, el segundo principio cooperativo, esto es, el relativo a la gestión democrática (García Álvarez, 2019, 17). Sin embargo, la formación cooperativa se extiende más allá de las fronteras de la sociedad cooperativa para poder llegar a toda la comunidad. En este sentido, desde una perspectiva externa, el quinto principio cooperativo debe orientarse al público en general para tener un impacto en el entorno social de la cooperativa (AAVV, 2012, 219 y Gaminde Egia, E. y Martínez Etxeberria, 2019, 107).

En lo que al contenido y alcance del principio se refiere, su análisis puede dividirse en tres partes, esto es: la educación; la formación; y la información. 
En primer lugar, la educación en el marco de las cooperativas está orientada a comprender el alcance del pensamiento cooperativo y el significado de las acciones cooperativas, ello en aras de construir una cultura organizacional y reforzar los valores cooperativos y el sentimiento de pertenencia a la organización. Pues bien, mediante el fortalecimiento de la identidad cooperativa y el reforzamiento del espíritu cooperativista, se pretende que las personas que forman parte de las cooperativas "sean plenamente conscientes de que su papel trasciende al de un trabajador de una empresa» (Rodríguez González, 2018, 9) y se conviertan en "ciudadanos más activos» (Martínez Charterina, 2020, 142). Así, se persigue que las personas que integran la organización no respondan solamente a los objetivos de la entidad, sino que también contribuyan a la consecución de los objetivos de la comunidad y de la sociedad en general. De este modo, la educación cooperativa contribuye a una realización más eficiente de las funciones por parte de los miembros de la misma, lo que repercute, al final, en la mejora de la innovación social de la entidad y de su competitividad (Rodríguez González, 2018, 16).

En segundo lugar, la formación en las cooperativas está relacionada con la capacitación profesional de todas las personas implicadas en la entidad (Gadea Soler, 2012, 14). Es decir, la formación en el ámbito de la cooperativa persigue que todas las personas integrantes de la misma tengan las habilidades y aptitudes necesarias para llevar a cabo sus responsabilidades de manera eficaz (Arnáez Arce, 2015, 190 y Rodríguez González, 2018, 18) y que, al mismo tiempo, "comprendan, asuman, interioricen y pongan en práctica el resto de principios cooperativos» (Arrieta Idiakez, 2020, 150). Precisamente, la formación continua es indispensable para adaptarse a los cambios dentro de las organizaciones, a las transformaciones de los puestos de trabajo, a la irrupción de las nuevas tecnologías, etcétera, de tal forma que «resulta imprescindible que exista una circulación fluida del conocimiento en el interior de la organización» (Marcuello Servós y Saz Gil, 2008, 64). Y, a este respecto, no solamente hacen falta conocimientos técnicos, sino que también es necesaria la adquisición de determinadas aptitudes, habilidades, destrezas y competencias, prestándose atención, de esta manera, al desarrollo integral de las personas que integran la cooperativa. En definitiva, mediante la formación continua, las personas implicadas en la organización pueden mejorar sus conocimientos y, además, su cualificación profesional, factor este último que puede y debe redundar en la mejora de sus condiciones laborales (Rodríguez González, 2018, 9).

$Y$, en tercer lugar, la información implica el deber de difundir y divulgar el alcance de los principios y valores cooperativos y de los bene- 
ficios de la cooperación, ello porque «la visualización ayuda a un mayor entendimiento del fenómeno cooperativo» (Rodríguez González, $2018,18)$. En este sentido, la proporción de información por parte de las cooperativas hacia el exterior pretende hacer ver en la comunidad y la sociedad la importante labor que desempeña la cooperativa y los valores en los que se fundamenta. Esta labor de información tiene un objetivo publicitario y promotor del cooperativismo, ello con el fin último de fomentar la constitución de sociedades cooperativas como alternativa empresarial. Dicho de otra manera, mediante la labor de información se pretende trasladar la filosofía del cooperativismo y su funcionamiento a la «ciudadanía receptora de sus servicios y receptora de sus productos» (Martínez Etxeberria, 2020, 193). Pero la información hacia el exterior no tiene solamente un propósito publicitario, sino que también está orientada a la mejora del entorno socio-económico y ambiental (Server Izquierdo, Marí Vidal y Lajara Camilleri, 2011, 99). Respecto del alcance de esta tercera faceta del quinto principio, la formulación de la misma da a entender que la información debe orientarse hacia el exterior de la cooperativa, esto es, al entorno social de la misma. Sin embargo, es importante tener presente que el derecho de información también debe tener un enfoque interno vinculado al segundo principio cooperativo relativo al control democrático de los miembros. Y es que, para adoptar decisiones orientadas a gestionar democráticamente la cooperativa, se necesita información transparente y conocimiento pleno (Macías Ruano, 2015, 9).

En síntesis, las acciones descritas, esto es, la educación, la formación y la información cooperativas, están relacionadas, principalmente, con el movimiento cooperativo, con la actividad diaria de las cooperativas y con el interés general de la comunidad. Y para materializar este principio, en el ámbito jurídico interno, la legislación española y las legislaciones de las Comunidades Autónomas contemplan la constitución en el seno de las cooperativas de un Fondo destinado a la educación, formación e información (Martínez Etxeberria, 2020, 192).

\subsection{El quinto principio cooperativo como instrumento para promover el trabajo decente}

En términos generales, la educación y la formación «cumplen con una función cardinal para la consecución de objetivos económicos y sociales» (Brú y Rosal, 2001, 95) y se conciben como una «fuente de mejora, tanto de la competitividad como de la productividad en las organizaciones» (Server Izquierdo, Marí Vidal y Lajara Camilleri, 2011, 99). 
En el ámbito cooperativo, «la preocupación por la formación y educación ha constituido un elemento diferenciador de las cooperativas frente al resto de sociedades empresariales» (Server Izquierdo, Marí Vidal y Lajara Camilleri, 2011, 99). Así, la educación constituye un «motor del desarrollo cooperativo» en tanto en cuanto «eleva el nivel de vida de la sociedad» (Martínez Charterina, 2020, 144).

De este modo, la educación, la formación y la información se convierten en presupuestos necesarios, entre otros propósitos, para: conocer y comprender el alcance de los derechos y las responsabilidades dentro de la cooperativa y la razón de ser de la organización, todo ello en aras de participar de manera activa en la gestión de la misma; apoyar el desarrollo integral de las personas que integran la entidad; favorecer la promoción personal y profesional de sus miembros; ayudar al mantenimiento de la estabilidad y la cualificación profesional; promover la igualdad de oportunidades; impulsar la innovación ( $y$, en especial, la innovación social); aumentar el valor y mejorar la competitividad; contribuir al desarrollo sostenible; y fomentar el progreso social.

Ciertamente, todas estas finalidades promovidas por el quinto principio cooperativo ayudan a la consecución del trabajo decente y de calidad previamente descrito.

Este planteamiento concuerda con lo estipulado en la Resolución sobre la formación y el desarrollo de los recursos humanos, adoptada por la Conferencia Internacional del Trabajo en 2002. En virtud de la citada Resolución, el desarrollo de los recursos humanos, la educación y la formación contribuyen a promover los intereses de las personas, las empresas, la economía y la sociedad; esto es, se conciben como un instrumento de progreso social. Además, se considera que la educación y la formación favorecen el desarrollo económico y ayudan a lograr el pleno empleo y a promover la inserción social; o, dicho de otra forma, se presentan como un instrumento económico y social. Más aún, se cree que la educación y la formación ayudan a las personas a tener acceso a un trabajo decente; es decir, la educación y la formación se vinculan con el concepto de trabajo decente y se conciben como instrumentos adecuados para conseguir su realización. Y la educación y la formación también se muestran como elementos necesarios y esenciales para aprovechar plenamente las oportunidades y enfrentarse con los retos que plantea esta evolución para las empresas, los trabajadores y los países; en otras palabras, se consideran herramientas necesarias para afrontar los cambios que plantea el mundo del trabajo.

En esta línea, el Informe elaborado por la Comisión Mundial sobre el Futuro del Trabajo "Trabajar para un futuro más prometedor», de 
2019, destaca la necesidad de invertir en las capacidades de las personas y, más concretamente, hace alusión a dos propósitos.

Por un lado, se insta a reconocer formalmente un derecho universal al aprendizaje permanente y a establecer un sistema eficaz de aprendizaje permanente. Así, se matiza que el aprendizaje permanente no abarca solamente las competencias para trabajar, sino que también comprende el desarrollo de las destrezas y aptitudes necesarias para participar en una sociedad democrática.

Y, por otro lado, se destaca la necesidad de apoyar a las personas en las transiciones. Ciertamente, se insiste en el hecho de que los cambios, acaecidos bien en la organización del trabajo bien como consecuencia de la irrupción de las nuevas tecnologías, son una constante en el mundo del trabajo. En este contexto, la Comisión Mundial sobre el Futuro del Trabajo señala que para aprovechar estas transformaciones y para garantizar transiciones justas en el mercado laboral, es necesario incidir en el aprendizaje permanente.

Igualmente, en el ámbito de la Unión Europea, es necesario destacar la Comunicación de la Comisión al Consejo, al Parlamento Europeo, al Comité Económico y Social y al Comité de las Regiones, titulada "Políticas sociales y de empleo — un marco para invertir en la calidad-» y adoptada en 2001, en la que se dispone que la educación y la formación son instrumentos necesarios para fomentar y garantizar la calidad en el empleo.

Asimismo, en el plano interno, resulta oportuno tener en consideración el Acuerdo del Consejo de Ministros, de 27 de julio de 2018, por el que se aprueba el Plan Director por un Trabajo Digno 2018-2019-2020. A pesar de que en el mismo no se hace alusión expresa a la promoción de las sociedades cooperativas, hay algunos aspectos importantes que merecen ser destacados. En concreto, en el mismo se puntualiza la necesidad de avanzar hacia un tipo de empresa que genera empleo de calidad y apuesta por la formación permanente, la conciliación de la vida personal, familiar y laboral, y la igualdad de oportunidades entre hombres y mujeres. Si bien no se menciona explícitamente la sociedad cooperativa como ejemplo, se cree que estas entidades pueden constituir el tipo de empresa apropiado para lograr dichos fines. Además, en el texto se señala que el mayor problema de las personas que no encuentran un empleo es la falta de formación para desempeñarlo, mientras que el principal obstáculo de quienes sí tienen la formación necesaria es la falta de estabilidad en el empleo. Claramente, esta realidad pone de manifiesto la importancia de la formación y su estrecha relación con la empleabilidad, al tiempo que pone en evidencia la precariedad a la que se 
enfrentan las personas trabajadoras y la necesidad de hacer realidad el trabajo decente.

Con base en las disposiciones comentadas, cabe concluir que la educación y la formación, así como la información, son premisas básicas para promover la calidad en el empleo y el trabajo decente. Por consiguiente, resultan indispensables sistemas de educación y formación apropiados en el ámbito cooperativo para garantizar el trabajo decente (Brú y Rosal, 2001, 95).

Así las cosas, el quinto principio cooperativo debe concebirse como una herramienta óptima para materializar el trabajo decente en el entorno cooperativo. En este sentido, es necesario que las entidades cooperativas impartan educación de calidad a todas las personas implicadas en la misma para que comprendan e interioricen la razón de ser del movimiento cooperativo y compartan sus valores y principios, de tal forma que se genere entre los mismos un sentimiento de pertenencia a la organización y se ejerzan las funciones laborales de manera eficiente. Al mismo tiempo, las cooperativas deben invertir en la formación permanente de sus miembros, un instrumento necesario para afrontar eficazmente las constantes transformaciones del mundo del trabajo. En esta labor, resulta necesario incidir no solamente en la adquisición de conocimientos teóricos y técnicos sino también en la adquisición de competencias laborales, esto es, conocimientos transversales y destrezas y aptitudes que permitan afrontar y solventar los problemas profesionales de manera autónoma y eficaz (Rodríguez González, 2018, 3). Y, además, es imprescindible que la educación y la formación cooperativas trasciendan las fronteras de la organización y tengan un impacto en la sociedad. Esta proyección hacia el exterior ayudará a fomentar el cooperativismo y a mejorar el nivel educativo del entorno donde la cooperativa desarrolla su actividad (Macías Ruano, 2015, 9 y Rodríguez González, 2018, 20).

Ahora bien, debe tenerse presente que para que el quinto principio cooperativo sea efectivo en la práctica, resulta indispensable que la educación, la formación y la información sean de calidad. Más aún, es importante que las acciones formativas no se limiten a ser meras formaciones puntuales y esporádicas, sino que deben ser acciones diseñadas con base en proyectos de formación continua que presten atención a las verdaderas necesidades de la cooperativa. Y para conseguir la efectividad de este quinto principio, sin duda alguna, las cooperativas deben invertir tiempo, recursos humanos, recursos materiales y recursos económicos (Rodríguez González, 2018, 20). 


\section{A modo de conclusión}

El déficit global de trabajo decente requiere la búsqueda urgente de estrategias eficaces para garantizar empleos de calidad. En el ámbito de la Economía Social, las cooperativas son entidades apropiadas para promover el trabajo decente, en especial, por su preocupación por las personas y por su gestión democrática. En concreto, en el seno de las cooperativas, el quinto principio centrado en la educación, la formación y la información debe constituir una herramienta esencial para fomentar el trabajo decente. Precisamente, la educación en valores cooperativos y la formación continua tanto de conocimientos técnicos como de competencias laborales son imprescindibles para garantizar la calidad en el empleo. Asimismo, la información hacia el exterior, mediante la cual se pretende propagar la idea del cooperativismo como alternativa empresarial, sirve para vincular el ámbito cooperativo con la promoción del trabajo decente. Ahora bien, para lograr que las cooperativas sean un referente del trabajo decente, se necesitan sistemas de educación y formación apropiados. Ello quiere decir que las formaciones puntuales no son suficientes para conseguir este objetivo y que se requieren programas sólidos de educación y formación continua que den respuesta a las verdaderas necesidades de la cooperativa y de su entorno.

\section{Bibliografía}

AAVV. 2012. Sens et pertinence de la coopération. Un défi d'éducation. Canadá: Groupe Fides.

ALCANTARA, L.C.S, SAMPAIO, C.A.C. \& URIARTE, L. 2018. «Experiencia Cooperativa de Mondragón: la educación cooperativa como un proceso de transformación social», CIRIEC-España, Revista de Economía Pública, Social y Cooperativa, 93, 181-209, DOl:10.7203/CIRIEC-E.93.9217.

ALFONSO SÁNCHEZ, R. 2015. "Los principios cooperativos como principios configuradores de la forma social cooperativa». En CIRIEC-España. Revista Jurídica, núm. 27.

ARNÁEZ ARCE, V.M. 2015. «Educación, formación e información: el compromiso cooperativo con la juventud». En Arnáez Arce, V.M. (Coord.). Difusión de los valores y principios cooperativos entre la juventud. Madrid: Dykinson.

ARNÁEZ ARCE, Vega María. 2018. «La alternativa cooperativa en la prestación de servicios públicos sostenibles». Boletín de la Asociación Internacional de Derecho Cooperativo, n. 52 (julio), 123-35. https://doi.org/10.18543/ baidc-52-2018pp123-135. 
ARRIETA IDIAKEZ, F.J. 2020. «Aspectos medioambientales a considerar en la formación de los socios cooperativistas». En Boletín de la Asociación Internacional de Derecho Cooperativo, núm. 57. DOI: https://doi.org/10.18543/ baidc-57-2020pp147-169

ATXABAL RADA, A. 2015. "Las cooperativas, empresas democráticas para los jóvenes». En Arnáez Arce, V.M. (Coord.). Difusión de los valores y principios cooperativos entre la juventud. Madrid: Dykinson.

BENGOETXEA ALKORTA, A. 2016. "Las cooperativas». En CIRIEC-España, Revista jurídica, núm. 29.

BORDAS MARTÍNEZ, J. y PINILLA GARCÍA, F.J. 2020. "¿Trabajo decente o trabajo indecente?». En Tendencias Sociales. Revista de Sociología, núm. 5.

BRÚ, E. y ROSAL, M.H. 2001. «Trabajo decente y la formación profesional en Centroamérica y República Dominicana». En Boletín Cinterfor: Boletín Técnico Interamericano de Formación Profesional, núm. 151.

CALDERÓN, B. y CALDERÓN, M.J. 2012. «La calidad del empleo de las entidades de la Economía Social en períodos de crisis». En Ekonomiaz, núm. 79.

CANALDA CRIADO, S. 2019. «El fomento del empleo decente y sostenible en cooperativas y sociedades laborales». En REVESCO: revista de estudios cooperativos, núm. 132.

CONFERENCIA INTERNACIONAL DEL TRABAJO 1999. Memoria del Director General: trabajo decente. Ginebra: Oficina Internacional del Trabajo.

CRUZ VILLALÓN, J. 2019. "La centralidad del trabajo digno en un nuevo modelo social». En Revista Internacional y Comparada de Relaciones Laborales y Derecho del Empleo, vol. 7, núm. 4.

EGGER, P. y SENGENBERGER, W. 2001. «Problemas y políticas del trabajo decente». En Boletín Cinterfor: Boletín Técnico Interamericano de Formación Profesional, núm. 151.

GADEA SOLER, E. 2012. «Delimitación del concepto de cooperativa: de los principios cooperativos a la responsabilidad social corporativa». En CIRIECEspaña, Revista jurídica, núm. 23.

GAMINDE EGIA, E. 2018. «El modelo cooperativo: mucho más que una alternativa ante la crisis». En Arnáez Arce, V.M. e Izquierdo Muciño, M.E. (Coords.). Fomento del cooperativismo como alternativa económica y social sostenible. Una visión de México y España. Madrid: Dikynson.

GAMINDE EGIA, E. y MARTÍNEZ ETXEBERRIA, G. 2019. «Training of cooperative values as a decisive element in new jobs to be created by $21^{\text {st }}$ Century cooperatives». En Boletín de la Asociación Internacional de Derecho Cooperativo, núm. 54. DOI: https://doi.org/10.18543/baidc-54-2019pp97-114

GARCÍA ÁLVAREZ, B. 2019. "Sobre la noción de interés social en las sociedades cooperativas y los principios cooperativos». En CIRIEC-España, Revista jurídica, núm. 34.

GARCÍA NINET, I. 2014. «Cooperativas de trabajo asociado, sociedades laborales y fomento del cooperativismo». En Revista General de Derecho del Trabajo y de la Seguridad Social, núm. 37.

GHAl, D. 2003. «Trabajo decente. Concepto e indicadores». En Revista Internacional del Trabajo, vol. 122, num. 2. 
GIL Y GIL, J.L. 2017. «Trabajo decente y reformas laborales». En Revista Derecho Social y Empresa, núm. 7.

GIL Y GIL, J.L. 2020. «El trabajo decente como objetivo de desarrollo sostenible». En Lex Social, vol. 10, núm. 1.

LÓPEZ RODRÍGUEZ, Josune. 2019. «La promoción De Empleos Verdes Y Decentes a través De Cooperativas». Boletín De La Asociación Internacional De Derecho Cooperativo, n. ${ }^{\circ} 54$ (julio), 115-29. https://doi.org/10.18543/ baidc-54-2019pp115-129.

LOZANO LARES, F. 2016. "La eficacia jurídica del concepto de trabajo decente». En Revista Internacional y Comparada de Relaciones Laborales y Derecho del Empleo, vol. 4, núm. 4.

MACÍAS RUANO, A.J. 2015. «El quinto principio internacional cooperativo: educación, formación e información. Proyección legislativa en España». En CIRIEC-España. Revista jurídica de economía social y cooperativa, núm. 27.

MARCUELLO SERVÓS, C. y SAZ GIL, M.I. 2008. "Los principios cooperativos facilitadores de la innovación: un modelo teórico». En REVESCO: revista de estudios cooperativos, núm. 94.

MARTÍNEZ CHARTERINA, A. 2013. "Cooperativismo y economía del bien común». Boletín de la Asociación Internacional de Derecho Cooperativo, núm. 47. DOI: https://doi.org/10.18543/baidc-47-2013pp185-198

MARTÍNEZ CHARTERINA, A. 2015a. "La cooperativa: empresa democrática y social». En Revista de Derecho, Empresa y Sociedad, núm. 7.

MARTÍNEZ CHARTERINA, A. 2015b. "Las cooperativas y su acción sobre la sociedad». En REVESCO: revista de estudios cooperativos, núm. 117.

MARTÍNEZ CHARTERINA, A. 2020. «El principio cooperativo de educación, formación e información desde una perspectiva histórica y doctrinal». Boletín de la Asociación Internacional de Derecho Cooperativo, núm. 57. DOI: https://doi.org/10.18543/baidc-57-2020pp133-145

MARTÍNEZ ETXEBERRIA, G. 2020. «El reforzamiento de la identidad cooperativa a través de la formación: un elemento a considerar por el movimiento cooperativo». Boletín de la Asociación Internacional de Derecho Cooperativo, núm. 57. DOI: https://doi.org/10.18543/baidc-57-2020pp171-205

MORGADO PANADERO, p. 2006. «La Economía Social y su marco legal». En Morgado Panadero, p. (Dir.) y Burgos Rosado, L. (Coord.). Economía Social y Cooperativismo. Valladolid: Lex Nova.

PÉREZ GONZÁLEZ, M. del C., \& VALIENTE PALMA, L. 2019. Aproximación al perfil y calidad del empleo generado por las sociedades cooperativas andaluzas. REVESCO. Revista De Estudios Cooperativos, 130, 122-148. https:// doi.org/10.5209/REVE.60989

RODGERS, G. 2002. «El trabajo decente como una meta para la economía global». En Boletín Cinterfor: Boletín Técnico Interamericano de Formación Profesional, núm. 15.

SALINAS RAMOS, F. 1995. "Notas para bucear en la identidad cooperativa». En REVESCO: revista de estudios cooperativos, núm. 61.

SÁNCHEZ ESPADA, J., MARTÍN LÓPEZ, S., BEL DURÁN, P., \& LEJARRIAGA PÉREZ DE LAS VACAS, G. 2018. Educación y formación en emprendimiento 
social: características y creación de valor social sostenible en proyectos de emprendimiento social. REVESCO. Revista De Estudios Cooperativos, 129, 16-38. https://doi.org/10.5209/REVE.62492

SERVER IZQUIERDO, R.J., MARI VIDAL, S. y LAJARA CAMILLERI, N. 2011. «La formación como factor de competitividad en las cooperativas agroalimentarias». En AECA: Revista de la Asociación Española de Contabilidad y Administración de Empresas, núm. 95. 


\section{Derechos de autor}

El Boletín de la Asociación Internacional de Derecho Cooperativo es una revista de acceso abierto lo que significa que es de libre acceso en su integridad inmediatamente después de la publicación de cada número. Se permite su lectura, la búsqueda, descarga, distribución y reutilización legal en cualquier tipo de soporte sólo para fines no comerciales y según lo previsto por la ley; sin la previa autorización de la Editorial (Universidad de Deusto) o el autor, siempre que la obra original sea debidamente citada (número, año, páginas y DOI si procede) y cualquier cambio en el original esté claramente indicado.

\section{Copyright}

The International Association of Cooperative Law Journal is an Open Access journal which means that it is free for full and immediate access, reading, search, download, distribution, and lawful reuse in any medium only for non-commercial purposes, without prior permission from the Publisher or the author; provided the original work is properly cited and any changes to the original are clearly indicated. 